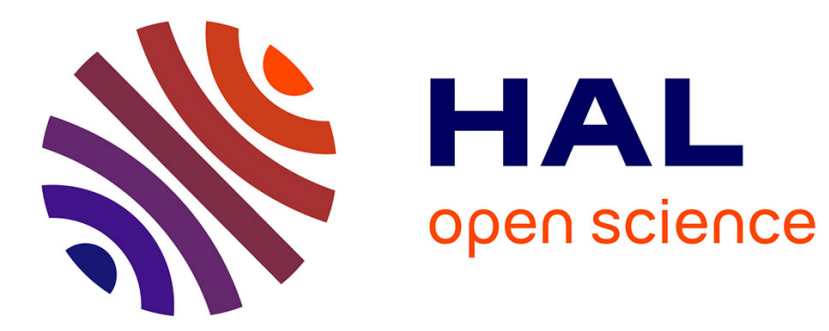

\title{
Walk Ratio: Perception of an Invariant Parameter of Human Walk on Virtual Characters
}

\author{
Benjamin Niay, Anne-Hélène Olivier, Katja Zibrek, Julien Pettré, Ludovic
}

Hoyet

\section{- To cite this version:}

Benjamin Niay, Anne-Hélène Olivier, Katja Zibrek, Julien Pettré, Ludovic Hoyet. Walk Ratio: Perception of an Invariant Parameter of Human Walk on Virtual Characters. SAP 2020 - ACM Symposium on Applied Perception, Sep 2020, Virtual Event, United States. pp.1-9, 10.1145/3385955.3407926 . hal-03009041

\section{HAL Id: hal-03009041 \\ https://hal.inria.fr/hal-03009041}

Submitted on 23 Nov 2020

HAL is a multi-disciplinary open access archive for the deposit and dissemination of scientific research documents, whether they are published or not. The documents may come from teaching and research institutions in France or abroad, or from public or private research centers.
L'archive ouverte pluridisciplinaire HAL, est destinée au dépôt et à la diffusion de documents scientifiques de niveau recherche, publiés ou non, émanant des établissements d'enseignement et de recherche français ou étrangers, des laboratoires publics ou privés. 


\section{Walk Ratio: Perception of an Invariant Parameter of Human Walk on Virtual Characters}

\author{
Benjamin Niay \\ benjamin.niay@inria.fr \\ Inria, Univ Rennes, CNRS, IRISA, \\ M2S, France
}

\author{
Anne-Hélène Olivier \\ anne-helene.olivier@irisa.fr \\ Inria, Univ Rennes, CNRS, IRISA, \\ M2S, France
}

\author{
Katja Zibrek \\ katja.zibrek@inria.fr \\ Inria, Univ Rennes, CNRS, IRISA, \\ M2S, France
}

\author{
Julien Pettré \\ julien.pettre@inria.fr \\ Inria, Univ Rennes, CNRS, IRISA, \\ M2S, France
}

Ludovic Hoyet

ludovic.hoyet@inria.fr

Inria, Univ Rennes, CNRS, IRISA,
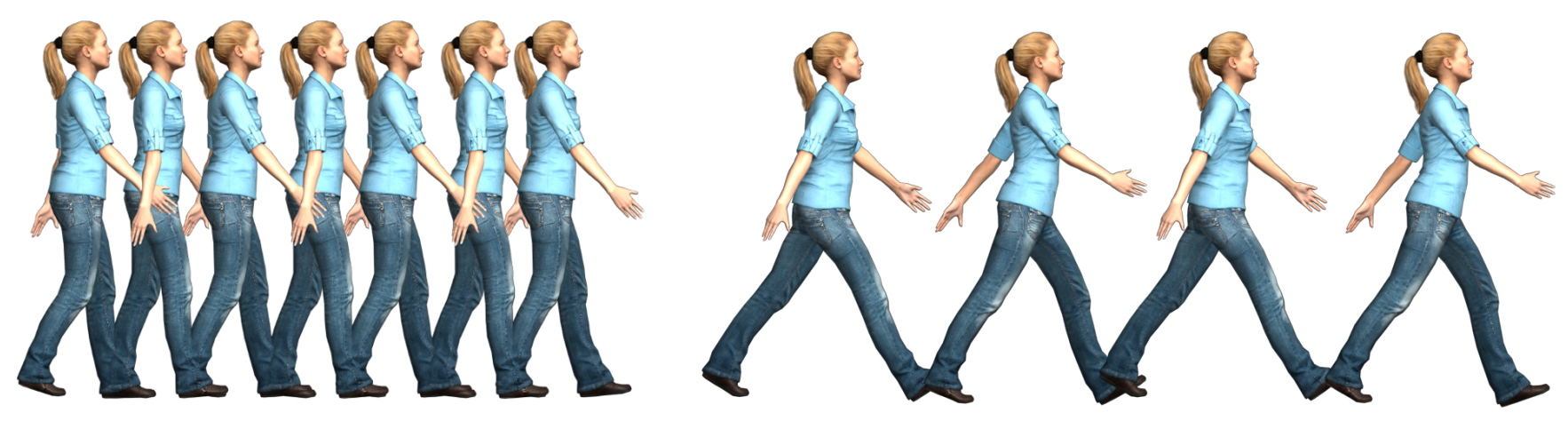

Figure 1: In this paper, we are interested in whether observers are able to recognize the natural Walk Ratio of an individual, an invariant parameter of human walking (ratio between step length and step frequency), when motions are displayed on virtual characters. In particular, the Walk Ratio represents the fact that different combinations of step length and step frequency can be selected by people to walk at a given speed, e.g., small steps at a high cadence (left) or longer steps at a lower cadence (right).

\begin{abstract}
Synthesizing motions that look realistic and diverse is a challenging task in animation. Therefore, a few generic walking motions are typically used when creating crowds of walking virtual characters, leading to a lack of variations as motions are not necessarily adapted to each and every virtual character's characteristics. While some attempts have been made to create variations, it appears necessary to identify the relevant parameters that influence users' perception of such variations to keep a good trade-off between computational costs and realism. In this paper, we therefore investigate the ability of viewers to identify an invariant parameter of human walking named the Walk Ratio (step length to step frequency ratio), which was shown to be specific to each individual and constant for different speeds, but which has never been used to drive animations of virtual characters. To this end, we captured 2 female and 2 male actors walking at different freely chosen speeds, as well as at different combinations of step frequency and step length. We then performed a perceptual study to identify the Walk Ratio that was perceived as
\end{abstract}

SAP '20, September 12-13, 2020, Virtual Event, USA

(C) 2020 Association for Computing Machinery.

This is the author's version of the work. It is posted here for your personal use. Not for redistribution. The definitive Version of Record was published in ACM Symposium on Applied Perception 2020 (SAP '20), September 12-13, 2020, Virtual Event, USA, https: //doi.org/10.1145/3385955.3407926. the most natural for each actor when animating a virtual character, and compared it to the Walk Ratio freely chosen by the actor during the motion capture session. We found that Walk Ratios chosen by observers were in the range of Walk Ratios measured in the literature, and that participants perceived differences between the Walk Ratios of animated male and female characters, as evidenced in the biomechanical literature. Our results provide new considerations to drive the animation of walking virtual characters using the Walk Ratio as a parameter, and might provide animators with novel means to control the walking speed of characters through simple parameters while retaining the naturalness of the locomotion.

\section{CCS CONCEPTS}

- Computing methodologies $\rightarrow$ Motion capture; Perception; - Human-centered computing $\rightarrow$ User studies.

\section{KEYWORDS}

Perception, Walk Ratio, Motion Capture, Animation, Virtual Human

\section{ACM Reference Format:}

Benjamin Niay, Anne-Hélène Olivier, Katja Zibrek, Julien Pettré, and Ludovic Hoyet. 2020. Walk Ratio: Perception of an Invariant Parameter of Human Walk on Virtual Characters. In ACM Symposium on Applied Perception 2020 (SAP '20), September 12-13, 2020, Virtual Event, USA. ACM, New York, NY, USA, 9 pages. https://doi.org/10.1145/3385955.3407926 


\section{INTRODUCTION}

Populating virtual environments with realistic virtual characters is a challenging task in animation and computer graphics. While improvement in overall realism has been achieved through motion capture and data-driven techniques when animating large groups of virtual humans, those still retain a certain uniformity in their motion. This is in part due to the fact that only a few motions are typically used, in combination with motion editing and time-warping techniques, e.g., to control the walking speed of the characters. However, adapting locomotion speed is more complex than only slowing-down or speeding-up animations [Pražák et al. 2010], as locomotion speed directly depends on the combination of step length and step frequency. While an almost infinite number of combinations of these parameters theoretically exists to produce a given walking speed, studies on human locomotion showed that each individual typically chooses a specific combination of these parameters to minimize energy expenditure [Zarrugh et al. 1974]. In particular, human walkers tend to keep a constant step length to step frequency ratio, which is specific to each individual and constant for different speeds [Sekiya et al. 1996]. This ratio, named the Walk Ratio, represents the relationship between amplitude and frequency of lower limb movement, and appears to present gender [Du Chatinier et al. 1970], age [Elbe et al. 1991; Gouelle et al. 2016], and morphology-related [Murray et al. 1966] differences.

As a first step to evaluate whether it is relevant to drive animations of virtual characters using this Walk Ratio as a control parameter, we explore in this paper the ability of observers to identify natural Walk Ratios. In particular, we are interested in whether observers are able to recognize a motion as biologically faithful by identifying the natural Walk Ratio of specific actors when their motions are applied on virtual characters. To this end, we motion captured 2 male and 2 female actors walking at different step frequencies and speeds. We then conducted a perceptual experiment to identify the ability of observers to detect the range of Walk Ratios considered as natural and compared it to the Walk Ratio freely chosen by actors when performing walks at the same speeds. We found that the perceived Walk Ratios were invariant across speeds while presenting gender differences, as evidenced in the biomechanical literature [Cho et al. 2004; Sekiya et al. 1996]. We also found that Walk Ratios chosen by observers from virtual character motions were in the range of Walk Ratios measured in the literature, despite being statistically different from the natural walk ratio of the actors. Interestingly, our results however highlighted differences in perceived Walk Ratios between male and female observers. Our results provide new considerations to drive the animation of walking virtual characters using the Walk Ratio as a control parameter, e.g., by enabling animators to control the speed of characters through simple parameters while retaining the naturalness of the locomotion.

The remaining of the article is organized as follows. Section 2 describes related work with respect to character animation techniques. Section 3 presents the general aim and hypotheses of the experiment, while Section 4 describes the experimental details. Section 5 presents the results, which are further discussed in Section 6. Section 7 provides the concluding remarks.

\section{RELATED WORK}

\subsection{Motion Editing}

Developments in motion capture systems and algorithms have undeniably improved the realism of virtual humans in the last decades. However, reusing, combining, sequencing, and editing captured motions is not trivial and has led to a large body of work in the Computer Animation community. For instance, a large family of approaches explored how to efficiently combine motion sequences. Rose et al. [1998] proposed to create verb and adverb graphs, where "verbs" are the motions, and "adverbs" the parameters that control them and which can be modified in real-time. Kovar et al. [2002a] proposed to organise motions in graphs, where transitions are created between similar poses and new motions synthesised by unrolling the motion graph. Similarly, Lee et al. [2002] proposed a search graph based on identifying plausible transitions between motion segments and clustering, while Arikan and Forsyth [2002] proposed to perform randomized search to identify the motions satisfying the most user constraints.

A large body of work also focuses on editing existing motions to satisfy a number of new constraints. Early works in motion editing techniques relied on tuning the signals of each degree of freedom (motion curves) to synthesise new motions. Witkins and Popović [1995] warped and blended motion curves to edit existing motions, while Bruderlin and Williams [1995] proposed a library of signal processing techniques to edit animations, including techniques such as filtering or motion interpolation with dynamic time warping. Le Callennec et al. [2006] also proposed a number of editing tools, including shape constraints and balance control, enabling animators to add significant changes to an animation while automatically satisfying a set of prioritized constraints. More recently, Holden et al. [2016] proposed editing techniques in the space of motion manifolds. Motion editing has also been used for correcting motion artefacts, e.g., footskate cleanup [Kovar et al. 2002b], physical implausibilities [Shin et al. 2003], or motion retargeting [Choi and Ko 2000; Gleicher 1998].

Another approach, called blending [Wiley and Hahn 1997], relies on interpolating similar motions (e.g. walking) to produce a new motion with interpolated time-varying weights from the example motions. It is one of the most commonly used techniques in animation, as it offers both realism of a motion capture dataset and control flexibility as long as the interpolating weights are adequate. Indeed the weights used for the interpolation can represent different animation parameters such as locomotion speed, heading direction, etc. Three main issues have to be addressed to successfully blend motions. First, motion clips have to be time-aligned, e.g., using time warping [Ahmed et al. 2001; Bruderlin and Williams 1995]. Second, the motions must be interpolated in a common representation space such as Euler, principle component [Glardon et al. 2004; Troje 2002] or Fourier [Unuma et al. 1995]. Lastly, the blended motion must be adapted to the virtual human morphology [Hecker et al. 2008; Kulpa et al. 2005; Multon et al. 2009]. Even though such techniques improved the usability of motion capture datasets, resulting motions might not be perceived as natural, as they for instance might not necessarily respect biomechanical parameters. 


\subsection{Motion Analysis}

Walking is one the most performed action by humans during their life. It is a cyclic locomotor task with invariant biomechanical properties among healthy humans. One of the most common metrics in gait analysis is walking speed which is a good indicator of functional ability and a predictor of future health status of a patient [Fritz and Lusardi 2009]. Walking speed can be defined as the product between step length (the length between two similar positions of opposite feet) and step frequency (the number of steps over a certain duration). Thus, it is theoretically possible to obtain an infinite combination of the two parameters to produce a given walking speed. However, humans are unlikely to adopt very long steps at very low frequencies or very short steps at very high frequencies to produce a given walking speed. More specifically, Sekiya et al. [1996] theorized that an invariant relationship exists between step length and step frequency for a range of speeds. They recorded these parameters and the walking speed of 64 participants and showed that the ratio between step length and frequency is invariant at different walking speeds for an individual, and that it corresponds to that individual's minimal energy expenditure when walking. While this ratio, named the Walk Ratio, shows invariance and reproducibility [Terrier and Schutz 2003], inter-individual differences have been pointed out. As reported by Hollman et al. [2011], age differences exist in the spatio-temporal parameters of walking between young adults and elderly. Indeed, elderly tend to reduce the length of their steps to obtain more stability and avoid falls [Elbe et al. 1991; Murray et al. 1966], which leads to a lower Walk Ratio. Gender differences also exist, where females tend to have a lower Walk Ratio than males, usually due to shorter step length in female than male walking motions [Cho et al. 2004].

While the Walk Ratio, computed from motion capture experiments, has already been investigated considering several factors (e.g., speed, gender, age) it remains to determine whether it is a salient visual feature of natural walking.

\subsection{Applied Perception}

The perception of biological body motion has also been a very active field of research for decades. Pioneer works from Johansson [1973] demonstrated that a few bright spots positioned on a person's body, and placed over a contrast background, were enough to be perceived as a human motion. Several researches built on these results and demonstrated that it is also possible to determine from such point light display stimuli gender from motion [Kozlowski and Cutting 1977; Troje 2002], or to recognize motions of a friend [Cutting and Kozlowski 1977]. Other researchers have also demonstrated that human visual perception is sensitive to invariant laws of motion. For instance, Dayan et al. [2007] demonstrated that human visual perception is influenced by stimuli complying with the twothirds power law, an invariant of human hand motion. Moreover, Polyakov [2009] suggested to use parabolic motion primitives as a control principle for arm movement, as they both respect the twothirds power law and minimize jerk. Similarly, Knopp et al. [2019] used motion primitives to generate walking animations and showed that such generated walking motions are not distinguishable from natural motions when applied to 3D virtual characters, through a perceptual study adapted from a Graphics Turing Test. It also has been shown that viewers can use motion cues to estimate the size of a walking creature [Jokisch and Troje 2003]. As virtual humans convey more information than point light display [Chaminade et al. 2007], studies in the Computer Graphics field are striving to access the perception of motion to improve realism of virtual characters' motions. For instance, previous work showed that both form and motion influence the perceived sex of virtual characters [McDonnell et al. 2009a; Zibrek et al. 2015], but that emotions are mostly conveyed by motion [McDonnell et al. 2009b].

Hoyet et al. [2013] investigated the distinctiveness and attractiveness of motions when applied to the same virtual character, e.g., to measure the ability of viewers to distinguish different actors performing the same motion when applied on a virtual character. Related to our work, their results indicate that viewers are able to differentiate between different actors' walking and running, which motivates our attempt at evaluating the ability of observers to identify natural Walk Ratios, an individual-specific invariant of motion, in walking virtual characters.

\section{OVERVIEW}

The general aim of this experiment is to investigate whether the Walk Ratio can be an interesting variable to generate realistic variability in virtual human animations of locomotion movements. More specifically, we would like to explore the ability of viewers to determine how natural different combinations of step length and step frequency are perceived when animating walking virtual characters. In particular, the ratio of these parameters, known as the Walk Ratio, is an invariant of human walk, which was shown to present gender and morphology differences [Hollman et al. 2011; Murray et al. 1966] . Our goal is therefore to investigate the ability of viewers to identify the Walk Ratio of specific actors when their motions are applied on virtual characters. To explore this issue, we conducted a perceptual experiment in which participants were asked to adjust the motions of virtual characters animated with motion capture data of 4 different actors walking at various step length and step frequency combinations. Walking speed was kept constant in each trial while participants adjusted the step frequency, leading to virtual characters walking with motions on a continuum from short steps with high step frequency, to long steps with low step frequency, therefore displaying a range of Walk Ratios. In particular, we expected that viewers would estimate a lower Walk Ratio for female than male actors, as differences were found according to the sex of the walker in the biomechanical literature [Cho et al. 2004; Sekiya et al. 1996]. We also expected viewers to estimate a similar Walk Ratio value at different speeds, as it was demonstrated to be invariant at different freely chosen walking speeds [Sekiya and Nagasaki 1998; Sekiya et al. 1997].

Thus, our main hypotheses are:

- H1: The Walk Ratio of motions displayed on virtual characters, as chosen by viewers as being the most natural, will be lower for female than for male actors.

- H2: The Walk Ratio of motions displayed on virtual characters, as chosen by viewers as being the most natural, will be invariant according to the walking speed. 
- H3: Viewers will be able to recognize the natural Walk Ratio for each individual actors when applied to their virtual counterpart.

\section{MATERIAL AND METHODS}

\subsection{Stimuli Preparation}

4.1.1 Motion Capture. For the purpose of the experiment, we captured the locomotion of 4 actors ( 2 males, 2 females) with no disability or neurological disorder that could affect their locomotion. Their height and age were respectively: F1 $(1.78 \mathrm{~m}, 26), \mathrm{F} 2(1.68 \mathrm{~m}$, $36), \mathrm{M} 1(1.80 \mathrm{~m}, 25), \mathrm{M} 2(1.78 \mathrm{~m}, 20)$. Motion capture was conducted using a Xsens IMU-based motion capture system at a sampling rate of $120 \mathrm{~Hz}$. We asked each actor to perform 15-meter straight walks according to different conditions. First, they were asked to walk at 5 different self-selected speeds (very slow, slow, comfort, fast and very fast). They always started with the comfort walking speed and could train as long as they wish for each speed before being recorded. No additional constraint was given, giving the choice of step length and step frequency to the actors. We then recorded a series of locomotion while varying step length and step frequency. Actors were instructed to adapt their step frequency to the sound of a metronome set from 80 steps per min (spm) to $120 \mathrm{spm}$ with a $10 \mathrm{spm}$ increase. For each frequency, we asked them to vary the length of their steps as following: very small, small, comfort, long and very long steps, resulting in 25 imposed walks at different step length and step frequency combinations. We decided to control step frequency primarily, as it has been demonstrated that it shows less variability than controlling step length in human walk [Sekiya et al. 1997], which is further discussed in Section 6.2.

For each of the 25 combinations we then created 1-cycle looping animations, using an approach similar to that described by Kovar et al. [2002a] and used by Hoyet et al. [2013]. In short, the approach automatically identifies from the original data the two most similar frames separated by a locomotion cycle, and linearly blends the difference between these two frames over the sequence, before removing any residual footsliding using the method of Kovar et al. [2002b].

4.1.2 Blend Tree. To control the walking animations, we designed a blend tree in Unity for each motion captured actor using the following procedure. After validating the exact step frequency of each 1-cycle walking motion, the motions of each actor were grouped according to their respective step frequency (i.e., 80, 90, 100, 110 or $120 \mathrm{spm}$ ) in 5 different sub-blend trees. Within each sub-blend tree, each motion was identified by its exact walking speed, and we used the desired walking speed as the blend parameter to interpolate between motions in the blend tree. Each sub-blend tree also included enough motions so that we could accurately generate motions with walking speeds in the range of 0.8 to $1.2 \mathrm{~m} / \mathrm{s}$. These 5 sub-blend trees were then controlled by a main blend tree which used the step frequency as a parameter to interpolate between the resulting motions (with the same speed) at different step frequencies. For instance, to obtain a walking animation at $1.0 \mathrm{~m} / \mathrm{s}$ and $85 \mathrm{spm}$, the 80 and 90 spm sub-blend trees would interpolate two of their respective walking motions to output a walking motion at $1.0 \mathrm{~m} / \mathrm{s}$ each, which would then be interpolated by the main blend tree

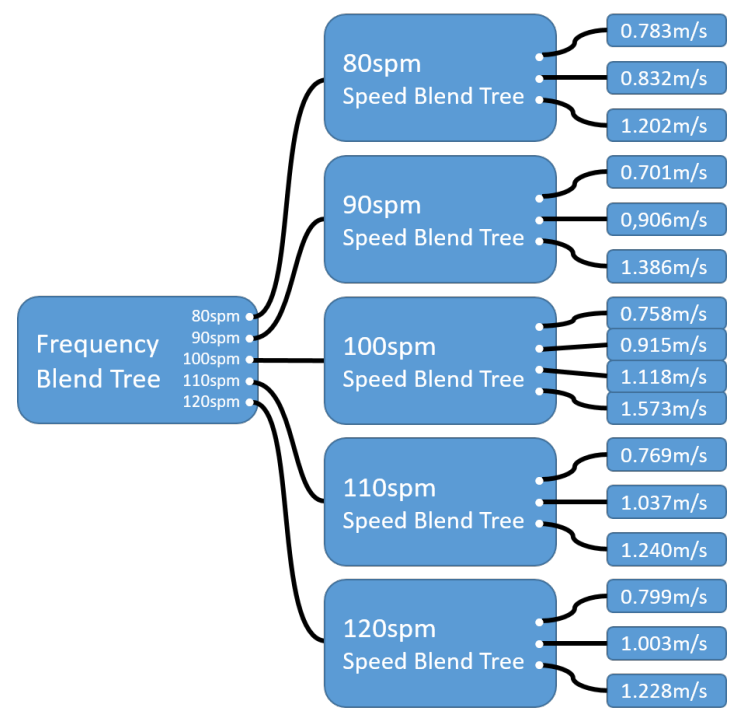

Figure 2: Example of blend tree of the actor F2, used to output a motion at a specific speed and step frequency (spm: steps per min)
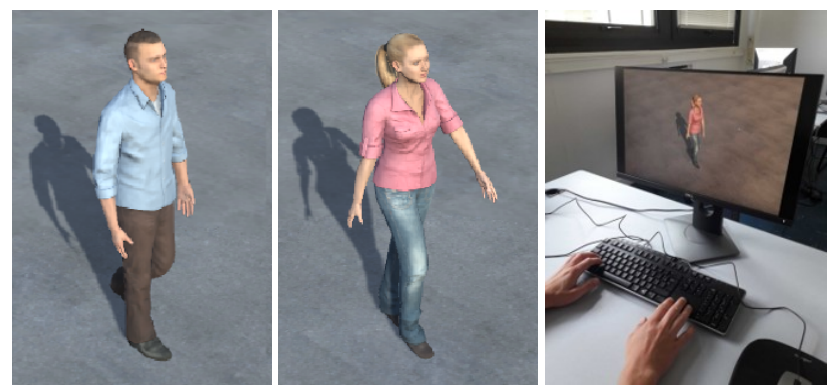

Figure 3: Male (Left) and Female (Center) character models used in our perceptual experiment. (Right) Perceptual experiment set up.

to generate a walking animation with the desired step frequency and speed. Figure 2 depicts as example the blend tree of one of the female actors. The blend trees were associated to 4 different virtual characters using 1 female and 1 male model (see Figure 3), which height were scaled according to each corresponding motion captured actor. Each interpolated motion of each actor was checked for speed, as reported in Table 1.

\subsection{Perceptual Experiment}

4.2.1 Participants. 22 Participants (10F and 12M) were recruited for the perceptual experiment. All participants volunteered for the experiment, and were recruited using internal mailing lists. Participants did not receive any compensation. They were all naive to the purpose of the experiment, and gave written and informed consent. The study conformed to the declaration of Helsinki. During the experience, they could ask for a break at any moment. At the 
Table 1: Interpolated speeds (mean \pm SD) from the blend trees at the different expected step frequencies and speeds, averaged over all four actors' motions.

\begin{tabular}{crccc}
\hline & \multicolumn{4}{c}{ Expected Speed (m/s) } \\
& & $\mathbf{0 . 8 0}$ & $\mathbf{1 . 0 0}$ & $\mathbf{1 . 2 0}$ \\
\hline Step & $\mathbf{8 0}$ & $0.801 \pm 0.006$ & $0.997 \pm 0.002$ & $1.198 \pm 0.002$ \\
Frequency & $\mathbf{1 0 0}$ & $0.798 \pm 0.001$ & $0.996 \pm 0.004$ & $1.198 \pm 0.004$ \\
(spm) & $\mathbf{1 1 0}$ & $0.798 \pm 0.006$ & $0.993 \pm 0.012$ & $1.192 \pm 0.008$ \\
& $\mathbf{1 2 0}$ & $0.816 \pm 0.027$ & $1.000 \pm 0.008$ & $1.203 \pm 0.006$ \\
\hline
\end{tabular}

end of the experience, a feedback was given on the objectives and the purpose of the experiment.

4.2.2 Task. Participants were instructed to modify the step frequency of the virtual characters. As speed is fixed by the blend tree, modifying the step frequency directly changes the associated Walk Ratio. We thus asked participants to modify continuously the step frequency of the virtual character presented on a 24-inch screen by pressing and holding the left and right arrow keys on a keyboard, until they found the motion to be most natural in their opinion. No information was given on what was a natural motion, but they were instructed that one key increased step frequency, while the other decreased it. As walking speed of the character was kept constant, changing the step frequency therefore led the blend tree to automatically produce the adequate combination of step frequency and step length for the corresponding speed. Participants were also first presented with two training stimuli which motions were not from the actors used in the experiment, depicting successively a high step frequency motion and a low step frequency motion, both at the same speed.

4.2.3 Experimental Design. Participants were presented with virtual characters displaying the motions of the 4 actors, and walking at 3 different walking speeds $(0.8,1.0$ and $1.2 \mathrm{~m} / \mathrm{s})$, using the blend trees described in Section 4.1.2. For each Actor $\times$ Speed combination, step frequencies were initialized once at a maximum of $120 \mathrm{spm}$, and once at a minimum of $80 \mathrm{spm}$ to avoid any effect of the initialisation value of the stimuli. In total 96 stimuli were presented in a random order to the participants: 4 Actors $\times 3$ Speeds $\times$ 2 Initializations $\times 4$ Repetitions. All the stimuli were displayed with the character walking in the middle of the screen using a typical canonical viewpoint (Figure 3), which is a viewpoint commonly used in video games and providing as much information as possible about the studied motions.

4.2.4 Analysis. From the perceptual study, as participants' answers corresponded to the motion they considered to be the most natural (i.e., a certain combination of step length and step frequency for a given speed), we computed the corresponding Walk Ratio perceived as the most natural by the observers. Statistical analysis was performed using the Jamovi software. Significant level was set at the $95 \%$ level, (i.e., $p<0.05$ ). Normality was assessed using Shapiro-Wilk test.

To assess whether the experimental factors affect how participants perceive the Walk Ratio, we first performed a 3-way repeated measure ANOVA with within-subject factors Actor, Speed and Initialization, and between-subject factor Participant Sex. GreenhouseGeisser adjustments to the degrees of freedom were applied when appropriate to avoid any violation of the sphericity assumption. Effect size was computed using partial eta squared $\left(\eta_{p}^{2}\right)$. Power analysis $(\beta)$ was performed from effect size, sample size and significance values using $G^{*}$ Power software. When needed, Tukey post-hoc tests were performed for pairwise comparisons. To evaluate the accuracy of participants to recognize each actor's Walk Ratio, we also performed a one sample Student t-test for each actor, comparing his/her Walk Ratio as estimated by participants on the corresponding character with his/her Walk Ratio computed from the walking motion captured at comfort speed (see Section 4.1.1).

\section{RESULTS}

Table 2 summarizes the effect of the experimental factors on the perceived Walk Ratio. The perceived Walk Ratio was highly affected by the Actor observed ( $\mathrm{p}<0.001, \eta_{p}^{2}=0.720, \beta=0.99$ ), where post-hoc analysis showed that on average the estimated Walk Ratio was significantly lower for female than male actors $(p<0.001$, see Figure 4, left). No difference was found between actors of the same sex. While we did not find an effect of Participant Sex $(p=0.736)$ on the perceived Walk Ratio, we found a moderate interaction effect between Actor and Participant Sex $\left(p=0.003, \eta_{p}^{2}=0.275\right)$. Post-hoc analysis showed that Female 1 was perceived as significantly different from Male 1 and Male 2 whatever the sex of the participant, while Female 2 was perceived as significantly different from Male 1 and Male 2 only by male participants, but not by female participants (see Figure 4, Center). This result therefore validates H1, while suggesting potential differences in the way male and female participants perceive natural Walk Ratios, which is discussed in Section 6.

Regarding walking speed, we did not find an effect of Speed on the perceived Walk Ratio $(\mathrm{p}=0.190)$. This result supports H2, and is in agreement with the biomechanical litterature where the measured Walk Ratio was found to be invariant at different freely chosen walking speeds [Sekiya and Nagasaki 1998; Sekiya et al. 1997]. However, a moderate interaction effect between Actor and Speed was observed ( $\mathrm{p}<0.001, \eta_{p}^{2}=0.295, \beta=0.99$ ), where posthoc analysis showed that the Walk Ratios of both female actors were perceived to be significantly lower than the Walk Ratios of both male actors for $1.0 \mathrm{~m} / \mathrm{s}$ and $1.2 \mathrm{~m} / \mathrm{s}$ speeds, but that only the Walk Ratio of Female 1 was perceived to be significantly lower than those of male actors for the speed of $0.8 \mathrm{~m} / \mathrm{s}$. In contrary, at $0.8 \mathrm{~m} / \mathrm{s}$, the Walk Ratio of Female 2 was not perceived to be significantly different from male actors, but perceived to be significantly different from Female 1 (see Figure 4 Right). There was however no effect of Initialisation ( $p=0.483$ ) on the perceived Walk Ratio, which suggests that participants perceived similar natural Walk Ratio independently of the initial step frequency of the motion displayed.

Finally, when comparing the perceived and captured Walk Ratio for each actor (where the perceived walk ratio of each actor was averaged over Speed for each participant, as we did not find a significant effect of Speed), results showed significant differences reported in Table 3. All the estimated Walk Ratios were smaller than the motion captured ones, except for Female 2, which was perceived 

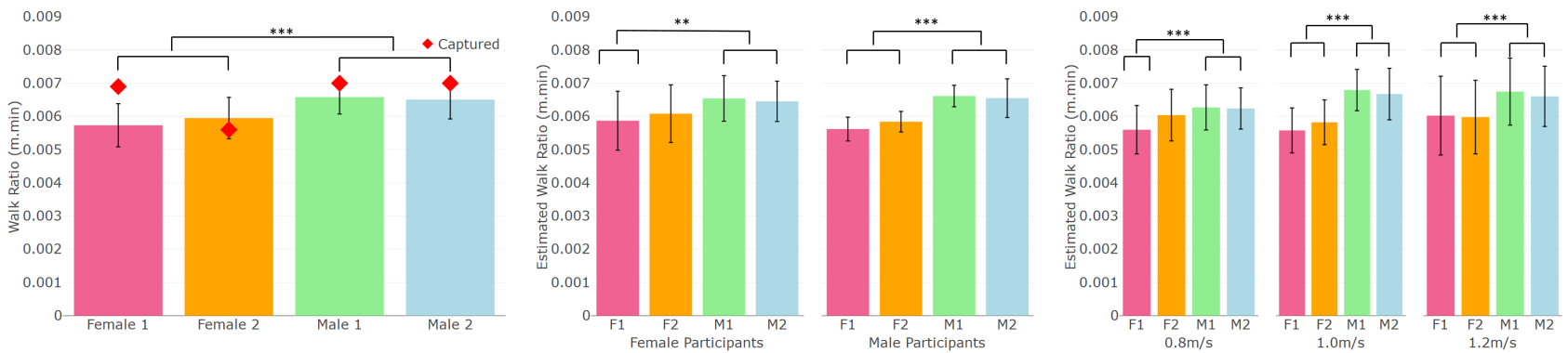

Figure 4: Main results of the experiment on the estimated Walk Ratio. (Left) main effect of Actor: the perceived Walk Ratio was significantly lower for female than male actors. The perceived Walk Ratios were estimated smaller than the captured Walk Ratio, depicted as red diamond, except for Female 2. (Center) interaction effect between Actor and Participant Sex. (Right) interaction effect between Actor and Speed.

Table 2: ANOVA results of the perceptual experiment. Our factors are Actors, Speed, Initialisation (Init.) and Participant Sex (P. Sex). Significant results are displayed in bold, or identified with a $\dagger$ when post-hoc analysis did not confirm the significance of the effect.

\begin{tabular}{llll}
\hline Factor & F-Test & p-value & $\eta^{2}$ \\
\hline Actor & $\mathbf{5 1 . 3 6 8}$ & $<\mathbf{0 . 0 0 1}$ & $\mathbf{0 . 7 2}$ \\
Speed & 1.729 & 0.19 & 0.08 \\
Init. & 0.512 & 0.483 & 0.025 \\
P. Sex & 0.117 & 0.736 & 0.006 \\
\hline Actor x Speed & $\mathbf{8 . 3 4 9}$ & $\mathbf{< 0 . 0 0 1}$ & $\mathbf{0 . 2 9 5}$ \\
Actor x Init & 12.715 & $<0.001 \dagger$ & 0.389 \\
Actor x P. Sex & $\mathbf{2 . 8 4 6}$ & $\mathbf{0 . 0 4 5}$ & $\mathbf{0 . 1 2 5}$ \\
Speed x Init & 6.706 & $0.003 \dagger$ & 0.251 \\
Speed x P. Sex & 2.392 & 0.104 & 0.107 \\
Init x P. Sex & 1.619 & 0.218 & 0.075 \\
\hline Actor x Speed x Init & 0.695 & 0.654 & 0.034 \\
Actor x Speed x P. Sex & 1.883 & 0.089 & 0.086 \\
Actor x Init x P. Sex & 1.27 & 0.293 & 0.06 \\
Speed x Init x P. Sex & 3.364 & $0.045 \dagger$ & 0.144 \\
\hline Actor x Speed x Init x P. Sex & 1.012 & 0.421 & 0.048 \\
\hline
\end{tabular}

to be higher (see Figure 4, Left). This result therefore invalidates H3, and suggests that viewers might not be able to recognize the actual natural Walk Ratio of an individual when applied to a virtual character, even though perceived Walk Ratios were in the range of those measured in the literature and relative differences between actors were indeed perceived.

\section{DISCUSSION}

In this paper, we presented a perceptual experiment evaluating whether viewers are able to identify the natural Walk Ratio specific to an individual, when captured motions are displayed on a virtual character.
Table 3: Motion captured and perceived Walk Ratios (m.min), with results of the one-sample student $t$-test comparing measured and perceived Walk ratios for each actor.

\begin{tabular}{lllll}
\hline Actor & Female 1 & Female 2 & Male 1 & Male 2 \\
\hline Captured & 0.00690 & 0.00560 & 0.00700 & 0.00700 \\
Estimated & 0.00573 & 0.00595 & 0.00658 & 0.00651 \\
SD & 0.00065 & 0.00062 & 0.00051 & 0.00058 \\
p-value & $<0.001$ & 0.015 & $<0.001$ & $<0.001$ \\
\hline
\end{tabular}

\subsection{Perception of the Walk Ratio}

First, we found that the natural Walk Ratio of the female actors was perceived to be significantly lower than the Walk Ratio of the male actors by the participants, which validates our hypothesis $\mathbf{H 1}$. This is the first time that this result, similar to what has been reported in the Movement Science literature about Walk Ratios measured on individuals actually walking [Cho et al. 2004; Sekiya et al. 1996], has been explored in relation to the perception of Walk Ratios. Furthermore, we found a slight difference in male and female participants' perceptions of the Walk Ratios regarding one female actor, where the perceived Walk Ratio of our Female 2 actor was not perceived to be significantly different from the Walk Ratios of male actors by female participants. While gender differences have been reported in the literature in the actual measurement of the Walk Ratio, there might also be gender differences in the way we perceive a natural Walk Ratio when animating virtual characters. As this is the first study evaluating the perception of this invariant parameter of human walking, it seems however difficult to explain the reason of the observed difference, especially given the number of actors used for the experiment. Further studies would therefore be required to investigate these effects, e.g., in a way similar to the work exploring gender differences in the perception of gender from motion [McDonnell et al. 2009a] or emotions [Zibrek et al. 2015].

Second, similarly to the literature which showed that measured Walk Ratios are relatively invariant at different freely chosen walking speeds (in the range of 0.6 to $2.2 \mathrm{~m} / \mathrm{s}$ ) [Murakami and Otaka 2017; Sekiya et al. 1996], we found that perceived Walk Ratios do 


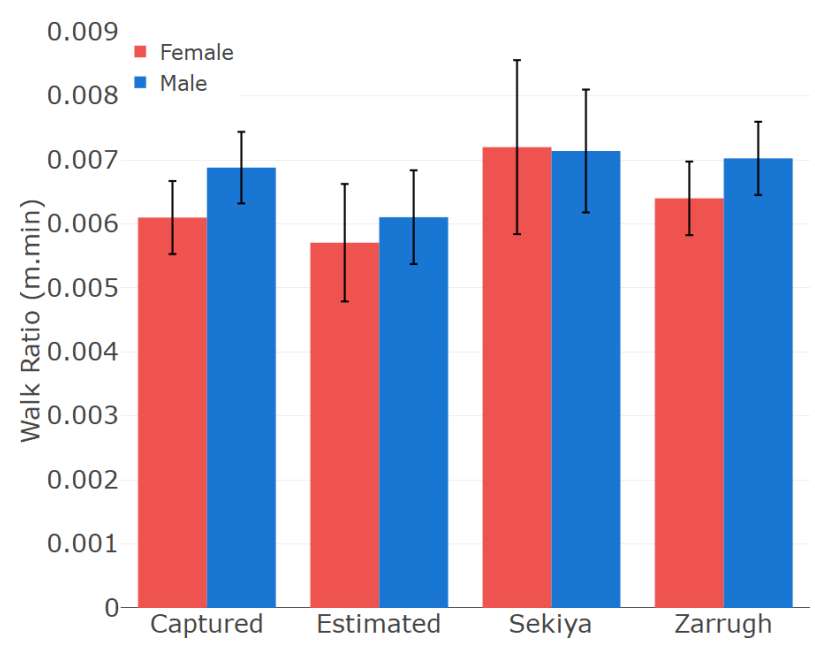

Figure 5: Estimated, Motion captured and Walk Ratios from Sekiya [1997], and Zarrugh [1974]

not seem to be influenced by the speed of the virtual character's motions, which validates our hypothesis $\mathbf{H} 2$. Our results however suggest that slight potential differences might still be perceived by viewers, as we found that the Walk Ratio of our Female actor 2 was perceived at a speed of $0.8 \mathrm{~m} / \mathrm{s}$ to be more similar to the Walk Ratios of our male actors than to the one of the other female actor.

It is also important to mention that the Walk Ratio has been studied in the Movement Science literature both in absolute [Borel et al. 2004; Menz et al. 2003; Sekiya and Nagasaki 1998] and normalized [Cho et al. 2004; Nagasaki et al. 1996; Sekiya et al. 1996] manners, where normalization is usually either performed based on the person's height or leg length to account for gender and height differences [Hof 1996]. When normalized, Walk Ratios tend do present less gender differences than Walk Ratios studied in absolute values, indicating that morphology might be more prevalent in the differences in Walk Ratios. However, for a first study exploring the ability of observers to identify natural Walk Ratio, we chose to analyze the data using absolute values only to possibly observe gender differences as measured on real persons [Cho et al. 2004]. This also seemed more appropriate given the small number of actors in our study, the lack of variability in actor's height, and the lack of consensus in normalization methods. However, future studies involving larger number of actors should investigate in more details the effect of actors' characteristics (e.g., height, gender, age) on the perceived Walk Ratio, exploring both absolute and normalized metrics when relevant. Moreover, while the effects of weight and Body Mass Index on various kinematic parameters during free walking have been widely studied [Lai et al. 2008; Silva-Hamu et al. 2013] there is, to our knowledge, no study investigating the effects of such factors on the Walk Ratio specifically. It would be thus interesting to get further insights on these aspects in the future.

Prior to the experiment, we also recorded our four actors walking at five different self-selected speeds, and measured their Walk Ratio from their self-selected step length and step frequency for each speed. The Walk Ratios we measured were in the range of those measured in the literature [Sekiya et al. 1997; Zarrugh et al. 1974] (see Figure 5). It is also interesting to point out that the Walk Ratios perceived as natural by the participants were in general lower than the motion captured Walk Ratios, except for Female 2, while also being in the same range than measured Walk Ratios found in the literature. This partially invalidates our hypothesis H3. To further understand these differences, it could therefore be interesting to explore experimental paradigms from studies performed in Virtual Reality, e.g., on misperceptions between real and perceived walking [Banton et al. 2005; Durgin et al. 2007]. However, such studies are usually performed in specific conditions, involving perception of self-motion from a first person perspective, and would therefore require to be adapted, e.g., to evaluate the perception of speed from a third person perspective or for non-player characters.

Finally, when biological walking motions are applied to virtual characters, our results demonstrate that the Walk Ratio perceived by observers has the same characteristics than the Walk Ratio naturally selected by walkers in real conditions. We therefore believe that these results can provide new considerations to drive the animation of walking virtual characters using the Walk Ratio as a parameter, which is our next goal to explore. For instance, we would like to propose novel animations techniques to edit the speed of existing walking motions by modifying concurrently step frequency and step length, while preserving an individual's characteristics by conserving the Walk Ratio of his/her original motion. It would also be interesting to explore how existing walking motions could be adapted to satisfy a novel Walk Ratio, so that the resulting motion would be perceived as corresponding to an individual with different characteristics (e.g., an older character).

\subsection{Limitations}

A limitation of our study is that walking motions were limited to the $0.8 \mathrm{~m} / \mathrm{s}$ to $1.2 \mathrm{~m} / \mathrm{s}$ speed range, while the human natural walking speed generally ranges from 1.2 to $1.6 \mathrm{~m} / \mathrm{s}$ [Kerrigan et al. 2001]. We purposely selected this range as we found in a pilot study that actors were able to comfortably follow the rhythm of the metronome when it was set between 80 and 120 spm, resulting in a range of speeds from 0.57 to $1.6 \mathrm{~m} / \mathrm{s}$. However, all the speeds were not obtained at all the step frequencies, which was required for completeness of the perceptual experiment. For example reaching $1.6 \mathrm{~m} / \mathrm{s}$ at $80 \mathrm{spm}$ would require actors to perform $1.3 \mathrm{~m}$ long steps. We therefore decided to select a speed range enabling us to obtain speeds that both respected the physical capabilities of our actors, and the invariability of the Walk Ratio [Murakami and Otaka 2017; Sekiya et al. 1996]. While the results in the range of speeds of our study indicate that participants perceived a similar Walk Ratio across speeds for a given actor, it would be interesting to further investigate the effect of higher walking speeds on the perception of the Walk Ratio. This would therefore also require to investigate these effects on a different range of step frequencies, more adapted to higher walking speeds.

One may also argue that we could have controlled step length over step frequency in the motion capture session. However, it has been demonstrated that controlling step frequency shows less variability than controlling step length in human walk [Sekiya 
et al. 1997], hence our choice. Adjusting the motions of the virtual characters in the perceptual experiment raised the same questioning. We decided to let participants adjust the motion using the step frequency as Durgin et al. [2007] explained that step frequency dominates over step length in the perception of self motion in a treadmill VR experiment.

Similarly, viewpoint might have affected the results of the experiment. We displayed the stimuli using a typical canonical viewpoint, as done by Prazak at al. [2011] and Hoyet et al. [2016]. In particular, this is a viewpoint typically used in the entertaining industry, especially in video-games. Furthermore, Jokisch and Troje [2006] demonstrated that viewers were better at recognising one's motion in half profile and frontal view in a point light display stimuli than in side view. However, it would be interesting to find out if the results remain similar in other viewpoints that can be found in interactive applications.

Another potential limitation of our study is that we decided to use linear interpolation (i.e., motion blending, a well know and widely used method in computer graphics) to produce the stimuli of our perceptual experiment. Studies demonstrated that averaged walking motions synthesized using linear interpolation were considered to be the most attractive when displayed amongst captured biological motions [2013]. Similarly, previous research explored the creation of visually appealing motion transitions using linear interpolation [Wang and Bodenheimer 2008], or of physically correct interpolated motions [Reitsma and Pollard 2003; Safonova and Hodgins 2005]. While it is a very popular method enabling to produce seamless and appealing motions when properly performed, little is however known about its effects on the perception of biological motion, and in the context of this study whether it might have an effect of the perception of Walk Ratios. It would therefore seem to be valuable for future studies to also investigate the consequences of synthesizing interpolated motions on motion perception.

While this is the first experiment exploring the perception of this invariant parameter of walking motions when displayed on virtual characters, and despite the fact that we found similarities between the perception of the Walk Ratio and actual measurements from the biomechanical literature, a last limitation of our study is the small number of male and female actors used. However, the current results make us believe that they should generalize to larger number of individuals. Still, we believe that more extensive studies need to be conducted in the future to further explore how subtle differences between individual walking motions are perceived, and how these elements can lever the creation of more realistic virtual character animations, such as for virtual crowds.

\section{CONCLUSION}

In this paper, we presented the first experiment studying the ability of viewer to identify the natural Walk Ratio of actors whose motions where applied to virtual characters. We designed a perceptual experiment in which participant were asked to adjust the motions of virtual characters until they found the walking motion the most natural. We found that participants visually identified Walk Ratios in the range of measured natural Walk Ratios, despite differences with the actual Walk Ratio of the actors. We also found some difference on how male and female participants perceive this invariant parameter of human walk. Our results provide new considerations to drive the animation of walking virtual characters using the Walk Ratio as a parameter, and might provide animators with novel means to control the speed of characters through simple parameters while retaining the naturalness of the locomotion.

\section{ACKNOWLEDGMENTS}

We wish to thank all the reviewers for their comments, and the participants in our experiments. This work was funded by the French ANR as part of the JCJC Per ${ }^{2}$ project (ANR-18-CE33-0013).

\section{REFERENCES}

Amr Ahmed, Farzin Mokhtarian, and Adrian Hilton. 2001. Parametric Motion Blending through Wavelet Analysis. In Proceedings of the 2001 ACM SIGGRAPH/Eurographics Symposium on Computer Animation. 7.

Okan Arikan and D. A. Forsyth. 2002. Interactive Motion Generation from Examples. ACM Trans. Graph. 21, 3 (July 2002), 483-490.

Tom Banton, Jeanine Stefanucci, Frank Durgin, Adam Fass, and Dennis Proffitt. 2005. The Perception of Walking Speed in a Virtual Environment. Presence: Teleoperators and Virtual Environments 14, 4 (2005), 394-406.

Liliane Borel, Françoise Harlay, Christophe Lopez, Jacques Magnan, André Chays, and Michel Lacour. 2004. Walking performance of vestibular-defective patients before and after unilateral vestibular neurotomy. Behavioural Brain Research 150, 1-2 (apr 2004), 191-200.

Armin Bruderlin and Lance Williams. 1995. Motion Signal Processing. In Proceedings of the 22Nd Annual Conference on Computer Graphics and Interactive Techniques (SIGGRAPH '95). ACM, New York, NY, USA, 97-104.

Benoit Le Callennec and Ronan Boulic. 2006. Interactive motion deformation with prioritized constraints. Graphical Models 68, 2 (2006), 175 - 193. Special Issue on SCA 2004.

Thierry Chaminade, Jessica Hodgins, and Mitsuo Kawato. 2007. Anthropomorphism influences perception of computer-animated characters' actions. Social Cognitive and Affective Neuroscience 2, 3 (05 2007), 206-216.

S.H. Cho, J.M. Park, and O.Y. Kwon. 2004. Gender differences in three dimensional gait analysis data from 98 healthy Korean adults. Clinical Biomechanics 19, 2 (Feb. 2004), 145-152.

Kwang-Jin Choi and Hyeong-Seok Ko. 2000. Online motion retargetting. The fournal of Visualization and Computer Animation 11, 5 (2000), 223-235.

James E. Cutting and Lynn T. Kozlowski. 1977. Recognizing friends by their walk: Gait perception without familiarity cues. Bulletin of the psychonomic society 9, 5 (1977), 353-356.

E. Dayan, A. Casile, N. Levit-Binnun, M. A. Giese, T. Hendler, and T. Flash. 2007. Neural representations of kinematic laws of motion: Evidence for action-perception coupling. Proceedings of the National Academy of Sciences 104, 51 (Dec. 2007), 20582-20587.

K Du Chatinier, NH Molen, and RH Rozendal. 1970. Step length, step frequency and temporal factors of the stride in normal human walking. Proceedings of the Koninklijke Nederlandse Akademie van Wetenschappen. Series C. Biological and medical sciences 73, 2 (1970), 214-227.

Frank H. Durgin, Catherine Reed, and Cara Tigue. 2007. Step Frequency and Perceived Self-motion. ACM Trans. Appl. Percept. 4, 1, Article 5 (Jan. 2007).

R.J. Elbe, S. Sienko Thomas, C. Higgins, and J. Colliver. 1991. Stride-dependant changes in gait of older people. Fournal of Neurology 238 (1991), 1-5.

Stacy Fritz and Michelle Lusardi. 2009. White paper:"walking speed: the sixth vital sign”. Journal of geriatric physical therapy 32, 2 (2009), 2-5.

Pascal Glardon, Ronan Boulic, and Daniel Thalmann. 2004. PCA-based walking engine using motion capture data. In Proceedings Computer Graphics International, 2004. 292-298.

Michael Gleicher. 1998. Retargetting Motion to New Characters. In Proceedings of the 25th Annual Conference on Computer Graphics and Interactive Techniques (SIGGRAPH '98). ACM, New York, NY, USA, 33-42.

Arnaud Gouelle, Julien Leroux, Jonathan Bredin, and Fabrice Mégrot. 2016. Changes in Gait Variability From First Steps to Adulthood: Normative Data for the Gait Variability Index. Fournal of Motor Behaviour 48, 3 (2016), 249-255.

Chris Hecker, Bernd Raabe, Ryan W. Enslow, John DeWeese, Jordan Maynard, and Kees van Prooijen. 2008. Real-time Motion Retargeting to Highly Varied User-created Morphologies. ACM Trans. Graph. 27, 3, Article 27 (Aug. 2008), 11 pages.

At L. Hof. 1996. Scaling gait data to body size. Gait \& Posture 4, 3 (1996), 222-223.

Daniel Holden, Jun Saito, and Taku Komura. 2016. A Deep Learning Framework for Character Motion Synthesis and Editing. ACM Trans. Graph. 35, 4, Article 138 (July 2016), 11 pages.

John H. Hollman, Eric M. McDade, and Ronald C. Petersen. 2011. Normative spatiotemporal gait parameters in older adults. Gait \& Posture 34, 1 (2011), 111 - 
118

Ludovic Hoyet, Anne-Hélène Olivier, Richard Kulpa, and Julien Pettré. 2016. Perceptual Effect of Shoulder Motions on Crowd Animations. ACM Transactions on Graphics 35, 4 (July 2016), 53:1-53:10.

Ludovic Hoyet, Kenneth Ryall, Katja Zibrek, Hwangpil Park, Jehee Lee, Jessica Hodgins, and O'Sullivan Carol. 2013. Evaluating the Distinctiveness and Attractiveness of Human Motions on Realistic Virtual Bodies. ACM Transactions on Graphics 32, 6 (Novembre 2013), 204:1-204:11.

Gunnar Johansson. 1973. Visual perception of biological motion and model for its analysis. Perception \& Psychophysics 14, 2 (1973), 201-211.

Daniel Jokisch, Irene Daum, and Nikolaus F Troje. 2006. Self Recognition versus Recognition of others by Biological Motion: Viewpoint-Dependent Effects. Perception 35, 7 (2006), 911-920.

Daniel Jokisch and Nikolaus F. Troje. 2003. Biological motion as a cue for the perception of size. Journal of Vision 3, 4 (05 2003), 252-264.

D.Casey Kerrigan, Laura W. Lee, James J. Collins, Patrick O. Riley, and Lewis A. Lipsitz 2001. Reduced hip extension during walking: Healthy elderly and fallers versus young adults. Archives of Physical Medicine and Rehabilitation 82, 1 (2001), 26 - 30

Benjamin Knopp, Dmytro Velychko, Johannes Dreibrodt, and Dominik Endres. 2019. Predicting Perceived Naturalness of Human Animations Based on Generative Movement Primitive Models. ACM Trans. Appl. Percept. 16, 3 (Sept. 2019), 1-18.

Lucas Kovar, Michael Gleicher, and Frédéric Pighin. 2002a. Motion Graphs. ACM Trans. Graph. 21, 3 (July 2002), 473-482.

Lucas Kovar, John Schreiner, and Michael Gleicher. 2002b. Footskate Cleanup for Motion Capture Editing. In Proceedings of the 2002 ACM SIGGRAPH/Eurographics Symposium on Computer Animation (San Antonio, Texas) (SCA '02). ACM, New York, NY, USA, 97-104.

Lynn T. Kozlowski and James E. Cutting. 1977. Recognizing the sex of a walker from a dynamic point-light display. Perception \& Psychophysics 21, 6 (1977), 575-580.

Richard Kulpa, Franck Multon, and Buno Arnaldi. 2005. Morphology-independent representation of motions for interactive human-like animation. Computer Graphics Forum 24, 3 (2005), 343-351.

Peggy P.K. Lai, Aaron K.L. Leung, Agnes N.M. Li, and M. Zhang. 2008. Threedimensional gait analysis of obese adults. Clinical Biomechanics 23 (Jan. 2008), S2-S6.

Jehee Lee, Jinxiang Chai, Paul S. A. Reitsma, Jessica K. Hodgins, and Nancy S. Pollard. 2002. Interactive Control of Avatars Animated with Human Motion Data. ACM Trans. Graph. 21, 3 (July 2002), 491-500.

Rachel McDonnell, Sophie Jörg, Jessica K. Hodgins, Fiona Newell, and Carol O'sullivan. 2009a. Evaluating the Effect of Motion and Body Shape on the Perceived Sex of Virtual Characters. ACM Trans. Appl. Percept. 5, 4, Article 20 (Feb. 2009), 14 pages.

Rachel McDonnell, Sophie Jörg, Joanna McHugh, Fiona N. Newell, and Carol O'Sullivan 2009b. Investigating the Role of Body Shape on the Perception of Emotion. ACM Trans. Appl. Percept. 6, 3, Article 14 (Sept. 2009), 11 pages.

Hylton B. Menz, Stephen R. Lord, and Richard C. Fitzpatrick. 2003. Acceleration patterns of the head and pelvis when walking on level and irregular surfaces. Gait \& Posture 18, 1 (aug 2003), 35-46.

Franck Multon, Richard Kulpa, Ludovic Hoyet, and Taku Komura. 2009. Interactive animation of virtual humans based on motion capture data. Computer Animation and Virtual Worlds 20, 5-6 (2009), 491-500.

Rumi Murakami and Yohei Otaka. 2017. Estimated lower speed boundary at which the walk ratio constancy is broken in healthy adults. Fournal of Physical Therapy Science 29, 4 (2017), 722-725.

M.P. Murray, R.C. Kory, B.H. Clarkson, and S.B. Sepic. 1966. COMPARISON OF FREE AND FAST SPEED WALKING PATTERNS OF NORMAL MEN. American fournal of Physical Medicine 45, 1 (1966), 8 - 24.

Hiroshi Nagasaki, Hajime Itoh, Ken Hashizume, Taketo Furuna, Hitoshi Maruyama, and Takashi Kinugasa. 1996. WALKING PATTERNS AND FINGER RHYTHM OF OLDER ADULTS. Perceptual and Motor Skills 82 (1996), 435-447.

Felix Polyakov, Eran Stark, Rotem Drori, Moshe Abeles, and Tamar Flash. 2009. Parabolic movement primitives and cortical states: merging optimality with geometric invariance. Biol Cybern 100, 2 (Feb. 2009), 159-184.

Martin Pražák, Ludovic Hoyet, and Carol O’Sullivan. 2011. Perceptual Evaluation of Footskate Cleanup. In Proceedings of the 2011 ACM SIGGRAPH/Eurographics Symposium on Computer Animation (Vancouver, British Columbia, Canada) (SCA '11). Association for Computing Machinery, New York, NY, USA, 287-294.

Martin Pražák, Rachel McDonnell, and Carol O'Sullivan. 2010. Perceptual Evaluation of Human Animation Timewarping. In ACM SIGGRAPH ASIA 2010 Sketches (Seoul, Republic of Korea) (SA '10). ACM, New York, NY, USA, Article 30, 2 pages.

Paul S. A. Reitsma and Nancy S. Pollard. 2003. Perceptual Metrics for Character Animation: Sensitivity to Errors in Ballistic Motion. In ACM SIGGRAPH 2003 Papers (San Diego, California) (SIGGRAPH '03). Association for Computing Machinery, New York, NY, USA, 537-542.

Charles Rose, Michael F. Cohen, and Bobby Bodenheimer. 1998. Verbs and adverbs: multidimensional motion interpolation. IEEE Computer Graphics and Applications 18, 5 (Sep. 1998), 32-40.

Alla Safonova and Jessica K. Hodgins. 2005. Analyzing the Physical Correctness of Interpolated Human Motion. In Proceedings of the 2005 ACM SIGGRAPH/Eurographics
Symposium on Computer Animation (Los Angeles, California) (SCA '05). Association for Computing Machinery, New York, NY, USA, 171-180.

Noboru Sekiya and Hiroshi Nagasaki. 1998. Reproducibility of the walking patterns of normal young adults: test-retest reliability of the walk ratio(step-length/step-rate). Gait \& Posture 7, 3 (May 1998), 225-227.

Noboru Sekiya, Hiroshi Nagasaki, Hajime Ito, and Taketo Furuna. 1997. Optimal Walking in Terms of Variability in Step Length. Fournal of Orthopaedic \& Sports Physical Therapy 26, 5 (1997), 266-272.

Noboru Sekiya, Hiroshi Nagasaki, Hajime Itoh, and Taketo Furuna. 1996. The invariant relationship between step length and step rate during free walking. fournal of Human Mouvement Studies 30 (1996), 241-257.

Hyun Joon Shin, Lucas Kovar, and Michael Gleicher. 2003. Physical touch-up of human motions. In 11th Pacific Conference onComputer Graphics and Applications, 2003. Proceedings. IEEE Comput. Soc, Canmore, Alta., Canada, 194-203.

Tânia Silva-Hamu, Cibelle Kayenne Martins Roberto Formiga, Flávia Martins Gervásio, Darlan Martins Ribeiro, Gustavo Christofoletti, and Jônatas de França Barros. 2013. The impact of obesity in the kinematic parameters of gait in young women. IfGM (June 2013), 507.

Philippe Terrier and Yves Schutz. 2003. Variability of gait patterns during unconstrained walking assessed by satellite positioning (GPS). European fournal of Applied Physiology 90, 5 (01 Nov 2003), 554-561.

Nikolaus. F. Troje. 2002. Decomposing biological motion: A framework for analysis and synthesis of human gait patterns. Fournal of Vision 2 (2002), 371-387.

Munetoshi Unuma, Ken Anjyo, and Ryozo Takeuchi. 1995. Fourier principles for emotion-based human figure animation. In Proceedings of the 22nd annual conference on Computer graphics and interactive techniques - SIGGRAPH '95. ACM Press, Not Known, 91-96.

Jing Wang and Bobby Bodenheimer. 2008. Synthesis and Evaluation of Linear Motion Transitions. ACM Trans. Graph. 27, 1, Article 1 (March 2008), 15 pages.

Douglas J. Wiley and James K. Hahn. 1997. Interpolation synthesis of articulated figure motion. IEEE Comput. Grap. Appl. 17, 6 (Dec. 1997), 39-45.

Andrew Witkin and Zoran Popovic. 1995. Motion Warping. In Proceedings of the 22Nd Annual Conference on Computer Graphics and Interactive Techniques (SIGGRAPH '95). ACM, New York, NY, USA, 105-108.

M. Y. Zarrugh, F. N. Todd, and H. J. Ralston. 1974. Optimization of energy expenditure during level walking. Europ. 7. Appl. Physiol. 33, 4 (1974), 293-306.

Katja Zibrek, Ludovic Hoyet, Kerstin Ruhland, and Rachel Mcdonnell. 2015. Exploring the Effect of Motion Type and Emotions on the Perception of Gender in Virtual Humans. ACM Trans. Appl. Percept. 12, 3, Article 11 (July 2015), 20 pages. 